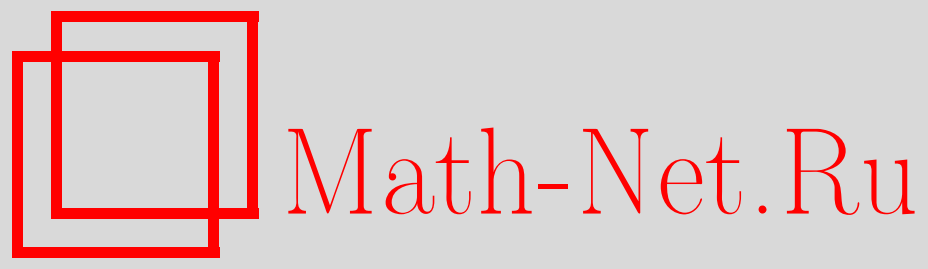

В. В. Басов, А. С. Ваганян, О нахождении неполной нормальной формы Белицкого гамильтоновой системы, Функи. анализ и его прил., 2014, том 48, выпуск 4, 9-18

DOI: https://doi.org/10.4213/faa3162

Использование Общероссийского математического портала MathNet.Ru подразумевает, что вы прочитали и согласны с пользовательским соглашением

http://www.mathnet.ru/rus/agreement

Параметры загрузки:

IP : 54.237 .59 .107

26 апреля 2023 г., 08:45:22

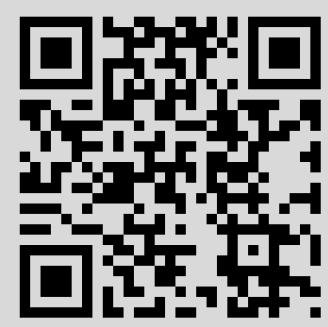




\title{
О нахождении неполной нормальной формы Белицкого гамильтоновой системы*
}

\author{
(C) 2014. В. В. БАСОВ, А. С. ВАГАНЯН
}

\begin{abstract}
Предложен эффективный метод нахождения неполной нормальной формы Белицкого гамильтоновой системы с многими степенями свободы с квазиоднородной невозмущенной частью специального вида. В частности, получены явные выражения для неполных нормальных форм Белицкого гамильтоновых систем с двумя степенями свободы с невозмущенным гамильтонианом вида $h_{1} x_{1}^{l_{1}} y_{1}^{m_{1}}+h_{2} x_{2}^{l_{2}} y_{2}^{m_{2}}$.
\end{abstract}

\section{§1. Введение}

При исследовании особой точки системы обыкновенных дифференциальных уравнений (ОДУ) часто вместо исходной системы рассматривают более простую, эквивалентную ей относительно выбранной группы локальных преобразований. Наиболее простые из получаемых таким образом систем называют нормальными формами.

В предлагаемой работе мы будем иметь дело с гамильтоновыми системами и каноническими преобразованиями, причем нас будет интересовать случай, когда линейная часть рассматриваемой системы вырождена, так как к нему неприменима классическая теория гамильтоновых нормальных форм, развитая Зигелем, Биркгофом, Брюно и их последователями. Один из наиболее универсальных подходов к определению нормальных форм таких систем был предложен Белицким в работах [1], [2]. Соответствующие (обобщенные) нормальные формы, называемые нормальными формами Белицкого, описываются неявно, и их вычисление очень трудоемко. Цель настоящей работы - предъявить эффективный алгоритм нахождения так называемой неполной нормальной формы Белицкого гамильтоновой системы с невозмущенной частью специального вида, позволяющий свести исходную задачу к задаче с меньшим числом степеней свободы. Отметим также, что мы будем рассматривать более общую ситуацию, чем Белицкий. Белицкий предполагал невозмущенную часть однородной, хотя на самом деле достаточно квазиоднородности с «правильным» весом (см. [3]).

Перед тем как определить нормальную форму Белицкого, введем несколько определений.

1.1. Квазиоднородные полиномы. Пусть $\mathbb{K}=\mathbb{R}$ или $\mathbb{C}$ - основное поле, $u=\left(u_{1}, \ldots, u_{n}\right)$ и $v=\left(v_{1}, \ldots, v_{n}\right)$, где $u_{i}, v_{i}$ - скалярные переменные, а $n \in \mathbb{N}$.

Положим $w=\left(w_{1}, \ldots, w_{2 n}\right)$, где $w_{1}=u_{1}, \ldots, w_{n}=u_{n}, w_{n+1}=v_{1}, \ldots, w_{2 n}=v_{n}$.

Обозначим через $\mathbb{Z}_{+}$множество целых неотрицательных чисел, а через $\mathbb{K}[w]$ и $\mathbb{K}[[w]]$ соответственно алгебры полиномов и формальных степенных рядов OT $w$.

*Работа второго автора выполнена при поддержке Лаборатории им. П. Л. Чебышева СПбГУ, грант Правительства РФ, дог. 11.G34.31.0026. 
Определение 1. Вектор $\gamma=\left(\gamma_{1}, \ldots, \gamma_{2 n}\right) \in \mathbb{N}^{2 n}$ называется весом переменной $w$, если НОД $\left(\gamma_{1}, \ldots, \gamma_{2 n}\right)=1$. Если к тому же $\gamma_{1}+\gamma_{n+1}=\cdots=\gamma_{n}+\gamma_{2 n}=\delta$ для некоторого натурального $\delta$, будем говорить, что $\gamma-$ канонический вес.

Присваивая переменной $w$ вес $\gamma$, мы задаем на $\mathbb{K}[w]$ и $\mathbb{K}[[w]]$ градуировку, в которой понятия степени, порядка и однородности заменяются соответственно понятиями обобщенной степени, обобщенного порядка и квазиоднородности. А именно, назовем обобщенной степенъю монома $w^{p}$ с весом $\gamma$ скалярное произведение $p \cdot \gamma$. Соответственно $P \in \mathbb{K}[w]$ назовем квазиоднородным полиномом обобщенной степени $k$ с весом $\gamma$ и обозначим через $P_{\gamma}^{[k]}$, если он является линейной комбинацией мономов обобщенной степени $k$ с весом $\gamma$. Переразлагая произвольный полином (формальный ряд) $P$ в сумму квазиоднородных слагаемых $P_{\gamma}^{[k]}$, определим его обобщенную степень $\operatorname{deg}_{\gamma} P$ (обобщенный порядок $\operatorname{ord}_{\gamma} P$ ) как максимальную (минимальную) из обобщенных степеней его слагаемых. При этом по соглашению $\operatorname{deg}_{\gamma} 0=0$ и $\operatorname{ord}_{\gamma} 0=\infty$.

Обозначим линейное пространство квазиоднородных полиномов от $w$ обобщенной степени $k$ с весом $\gamma$ через $\mathbb{K}_{\gamma}^{[k]}$.

Условие каноничности веса означает, что скобка Пуассона квазиоднородных полиномов квазиоднородна, т. е. $\left\{P_{\gamma}^{[k]}, Q_{\gamma}^{[l]}\right\} \in \mathbb{K}_{\gamma}^{[k+l-\delta]}$.

Для всякого $Q \in \mathbb{K}[w]$ положим $Q^{*}=\bar{Q}(\partial)$, где верхняя черта означает комплексное сопряжение коэффициентов, а $\partial=\left(\partial / \partial w_{1}, \ldots, \partial / \partial w_{2 n}\right)$. Тогда формула

$$
\langle\langle P, Q\rangle\rangle=\left.P(\partial) \bar{Q}(w)\right|_{w=0} \quad(P, Q \in \mathbb{K}[w])
$$

определяет на $\mathbb{K}[w]$ скалярное произведение со следующими свойствами:

(i) $\left\langle\left\langle w^{\alpha}, w^{\beta}\right\rangle\right\rangle=\alpha ! \delta_{\alpha \beta}$,

(ii) $\langle\langle P Q, R\rangle\rangle=\left\langle\left\langle P, Q^{*} R\right\rangle\right\rangle(P, Q, R \in \mathbb{K}[w])$, где $\alpha$ ! $=\alpha_{1} ! \cdots \alpha_{2 n}$ !, а $\delta_{\alpha \beta}=\prod_{i=1}^{2 n} \delta_{\alpha_{i} \beta_{i}}-$ произведение символов Кронекера.

Из свойства (i) следует, что $\mathbb{K}[w]$ разбивается в прямую сумму ортогональных подпространств $\mathbb{K}_{\gamma}^{[k]}$ квазиоднородных полиномов обобщенной степени $k \mathrm{c}$ весом $\gamma$.

А по свойству (ii) $Q^{*}$ - это оператор, сопряженный умножению на $Q$ относительно $\langle\langle\cdot, \cdot\rangle\rangle$.

1.2. Резонансное уравнение и определение неполной нормальной формы Белицкого. Пусть даны канонический вес $\gamma$ и гамильтониан

$$
H(w)=H_{\gamma}^{[\chi]}(w)+V(w)
$$

с невозмущенной частью $H_{\gamma}^{[\chi]} \in \mathbb{K}_{\gamma}^{[\chi]}$ и возмущением $V \in \mathbb{K}[[w]]$, такими, что $\chi \geqslant \delta=\gamma_{i}+\gamma_{n+i}, H_{\gamma}^{[\chi]} \not \equiv 0$ и $\operatorname{ord}_{\gamma} V>\chi$.

Рассмотрим оператор $\widehat{H}_{\gamma}^{[\chi]}{ }^{*}$ на $\mathbb{K}[w]$, сопряженный векторному полю $\widehat{H}_{\gamma}^{[\chi]}=$ $\left\{H_{\gamma}^{[\chi]}, \cdot\right\}$ :

$$
\left\langle\left\langle\widehat{H}_{\gamma}^{[\chi]}(P), Q\right\rangle\right\rangle=\left\langle\left\langle P, \widehat{H}_{\gamma}^{[\chi]^{*}}(Q)\right\rangle\right\rangle
$$


По свойству (ii) скалярного произведения $\langle\cdot \cdot, \cdot\rangle$ имеют место следующие эквивалентные формулы для $\widehat{H}_{\gamma}^{[\chi]^{*}}$ :

$$
\begin{aligned}
\widehat{H}_{\gamma}^{[\chi]^{*}} & =\sum_{i=1}^{n} w_{n+i}\left(\frac{\partial H_{\gamma}^{[\chi]}}{\partial w_{i}}\right)^{*}-w_{i}\left(\frac{\partial H_{\gamma}^{[\chi]}}{\partial w_{n+i}}\right)^{*}, \\
\widehat{H}_{\gamma}^{[\chi]^{*}} & =\sum_{i=1}^{n}\left(\frac{\partial H_{\gamma}^{[\chi]}}{\partial w_{i}}\right)^{*} w_{n+i}-\left(\frac{\partial H_{\gamma}^{[\chi]}}{\partial w_{n+i}}\right)^{*} w_{i} .
\end{aligned}
$$

Определение 2. Резонансным уравнением для $H_{\gamma}^{[\chi]}$ назовем уравнение

$$
\widehat{H}_{\gamma}^{[\chi]^{*}}(P)=0 \quad(P \in \mathbb{K}[w]) .
$$

Линейное пространство $\mathfrak{R}_{\gamma}$ его решений назовем пространством резонансных полиномов.

Из квазиоднородности полинома $H_{\gamma}^{[\chi]}$ вытекает, что $\mathfrak{R}_{\gamma}$ разбивается в прямую сумму ортогональных линейных подпространств $\mathfrak{R}_{\gamma}^{[k]}$ резонансных квазиоднородных полиномов обобщенной степени $k \in \mathbb{Z}_{+}$с каноническим весом $\gamma$.

Определение 3 [1]. Гамильтониан (1) называется неполной нормальной формой Белицкого, если его возмущение состоит из резонансных полиномов, т. е. $V_{\gamma}^{[k]} \in \mathfrak{R}_{\gamma}^{[k]}$ для всех $k \in \mathbb{Z}_{+}$.

Таким образом, нахождение неполной нормальной формы Белицкого заключается в решении резонансного уравнения. Сам Белицкий в своих работах не предлагает для этого никаких методов, а лежащие на поверхности методы линейной алгебры оказываются малоэффективными уже при $n=2$. В 33 мы покажем, как можно обойти вычислительные трудности в случае невозмущенной части специального вида, а пока проиллюстрируем введенные определения в случае $n=1$.

Отметим, что произвольный гамильтониан (1) приводится к неполной нормальной форме Белицкого при помощи почти тождественного формального канонического преобразования (см. [3] или в однородном случае [1]).

Пример 1 [3, теорема 6]. Пусть $H_{\gamma}^{[\chi]}(w)=v^{m}, \chi=m \geqslant 2, \gamma=(1,1)$. Согласно $\left(22_{1}\right), \widehat{H}_{\gamma}^{[\chi]^{*}}=-u\left(m v^{m-1}\right)^{*}=-m u \partial^{m-1} / \partial v^{m-1} ;$ поэтому резонансное уравнение (3) имеет вид $\partial^{m-1} P / \partial v^{m-1}=0$, а неполная нормальная форма Белицкого - вид

$$
\widetilde{H}(z)=y^{m}+\sum_{k=m+1}^{\infty} \sum_{q=0}^{m-2} \widetilde{h}^{[k-q, q]} x^{k-q} y^{q}
$$

Пример 2 [3, теорема 7]. Пусть $H_{\gamma}^{[\chi]}(w)=u^{l} v^{m}, l, m \geqslant 1, d=$ НОД $(l, m)$, $\chi=l+m, \gamma=(1,1)$. Согласно $\left(2_{2}\right)$,

$$
\widehat{H}_{\gamma}^{[\chi]^{*}}=\left(l u^{l-1} v^{m}\right)^{*} v-\left(m u^{l} v^{m-1}\right)^{*} u=\frac{\partial^{l+m-2}}{\partial u^{l-1} \partial v^{m-1}}(l(\partial / \partial v) v-m(\partial / \partial u) u) .
$$

Следовательно, резонансное уравнение (3) имеет вид

$$
\frac{\partial^{l+m-2}}{\partial u^{l-1} \partial v^{m-1}}\left(l \frac{\partial(v P)}{\partial v}-m \frac{\partial(u P)}{\partial u}\right)=0
$$


а соответствующая неполная нормальная форма Белицкого - вид

$$
\begin{gathered}
\widetilde{H}(z)=x^{l} y^{m}+\sum_{k=l+m+1}^{\infty}\left(\sum_{p=0}^{l-2} \widetilde{h}^{[p, k-p]} x^{p} y^{k-p}+x^{l-1} \sum_{q=0}^{m-2} \widetilde{h}^{[k+l-1-q, q]} x^{k-q} y^{q}\right) \\
+\sum_{j=d+1+\left[\frac{3 d-1}{l+m}\right]}^{\infty} \widetilde{h}^{[j l / d-1, j m / d-1]} x^{j l / d-1} y^{j m / d-1} .
\end{gathered}
$$

На коэффициенты $\widetilde{h}$ не накладывается никаких ограничений, т. е. независимо от их значений гамильтониан $\widetilde{H}$ является неполной нормальной формой Белицкого.

\section{§2. Квазирезонансные полиномы}

Теорема 1. Пусть гамильтониан (1) таков, что $\chi>\delta$. Тогда $\mathbb{K}[w]$ единственным образом разбивается в прямую сумму по $j \in \mathbb{Z}_{+}$взаимно ортогональных линейных подпространств $\mathfrak{Q}_{\gamma, j}$, таких, что для кажсдого ненулевого $Q \in \mathfrak{Q}_{\gamma, j}$

$$
\widehat{H}_{\gamma}^{[\chi]}{ }^{* j}(Q) \neq 0, \quad \widehat{H}_{\gamma}^{[\chi]}{ }^{* j+1}(Q)=0
$$

где показатели $j$ и $j+1$ означают, что дифференциалъный оператор $\widehat{H}_{\gamma}^{[\chi]}{ }^{*}$ применяется $\kappa Q$ соответственно $j u j+1$ раз.

Доказательство. Существование разбиения. Пусть $k \in \mathbb{Z}_{+}$и $r_{k}=[k /(\chi-\delta)]$.

Определим линейные пространства $\mathfrak{H}_{\gamma, j}^{[k]}$ и $\mathfrak{Q}_{\gamma, j}^{[k]}\left(j=0, \ldots, r_{k}\right)$ следующим образом:

$\mathfrak{H}_{\gamma, j}^{[k]}=\widehat{H}_{\gamma}^{[\chi]}{ }^{j}\left(\mathbb{K}_{\gamma}^{[k-j(\chi-\delta)]}\right), \quad \mathfrak{Q}_{\gamma, j}^{[k]}=\left(\mathfrak{H}_{\gamma, j+1}^{[k]}\right)_{\mathfrak{H}_{\gamma, j}^{[k]}}^{\perp} \quad\left(\mathfrak{H}_{\gamma, j+1}^{[k]} \subseteq \mathfrak{H}_{\gamma, j}^{[k]}, \mathfrak{H}_{\gamma, r_{k}+1}^{[k]}=\{0\}\right)$.

В частности, $\mathfrak{H}_{\gamma, 0}^{[k]}=\mathbb{K}_{\gamma}^{[k]}, \mathfrak{Q}_{\gamma, 0}^{[k]}=\mathfrak{R}_{\gamma}^{[k]}, \mathfrak{Q}_{\gamma, r_{k}}^{[k]}=\mathfrak{H}_{\gamma, r_{k}}^{[k]}$.

Согласно $\left(5_{2}\right)$, пространства $\mathfrak{Q}_{\gamma, j}^{[k]}$ взаимно ортогональны и $\bigoplus_{j=0}^{r_{k}} \mathfrak{Q}_{\gamma, j}^{[k]}=\mathfrak{H}_{\gamma, 0}^{[k]}$ $=\mathbb{K}_{\gamma}^{[k]}$.

Пусть $Q \neq 0$ и $Q \in \mathfrak{Q}_{\gamma, j}^{[k]}$. Поскольку $\mathfrak{Q}_{\gamma, j}^{[k]} \subseteq \mathfrak{H}_{\gamma, j}^{[k]}, Q$ лежит в образе оператора $\widehat{H}_{\gamma}^{[\chi]^{j}}$. Пересечение этого образа с ядром оператора $\widehat{H}_{\gamma}^{[\chi]^{* j}}$ тривиально, а значит, $Q$ удовлетворяет соотношению $\left(4_{1}\right)$. С другой стороны, $Q \perp \mathfrak{H}_{\gamma, j+1}^{[k]}$, т. е. $\left\langle\left\langle Q, \widehat{H}_{\gamma}^{[\chi]}{ }^{j+1}(P)\right\rangle\right\rangle=\left\langle\left\langle\widehat{H}_{\gamma}^{[\chi]}{ }^{* j+1}(Q), P\right\rangle\right\rangle=0$ для каждого $P \in \mathbb{K}_{\gamma}^{[k-(j+1)(\chi-\delta)]}$, что эквивалентно равенству $\left(4_{2}\right)$.

Таким образом, полагая $\mathfrak{Q}_{\gamma, j}^{[k]}=\{0\}$ для $j>r_{k}$ и $\mathfrak{Q}_{\gamma, j}=\bigoplus_{k=0}^{\infty} \mathfrak{Q}_{\gamma, j}^{[k]}$, с учетом (5) получаем требуемое разбиение.

Единственность разбиения. Пусть $\bigoplus_{j=0}^{\infty} \mathfrak{Q}_{\gamma, j}=\bigoplus_{j=0}^{\infty} \widetilde{\mathfrak{Q}}_{\gamma, j}=\mathbb{K}[w]-$ два разбиения, удовлетворяющих условию теоремы. Индукцией по $j \in \mathbb{Z}_{+}$покажем, что $\mathfrak{Q}_{\gamma, j}=\widetilde{\mathfrak{Q}}_{\gamma, j}$. 
Пусть $Q \in \mathfrak{Q}_{\gamma, j}$. Тогда $Q$ единственным образом представляется в виде $Q=$ $\sum_{i=0}^{\infty} Q_{i}$, где $Q_{i} \in \widetilde{\mathfrak{Q}}_{\gamma, i}$. Из (4) получаем, что $Q_{j} \neq 0$, а $Q_{i}$ с $i>j$ равны нулю. Следовательно, если $j=0$, то $Q=Q_{0}$, что дает базу индукции: $\mathfrak{Q}_{\gamma, 0}=\widetilde{\mathfrak{Q}}_{\gamma, 0}$.

Пусть $\mathfrak{Q}_{\gamma, i}=\widetilde{\mathfrak{Q}}_{\gamma, i}$ для $i=0, \ldots, j-1, j \in \mathbb{N}$. Тогда $Q-Q_{j} \in \bigoplus_{i=0}^{j-1} \mathfrak{Q}_{\gamma, i}$. Отсюда

$$
\left\langle\left\langle Q-Q_{j}, Q-Q_{j}\right\rangle\right\rangle=\left\langle\left\langle Q-Q_{j}, \sum_{i=0}^{j-1} Q_{i}\right\rangle\right\rangle=\sum_{i=0}^{j-1}\left(\left\langle\left\langle Q, Q_{i}\right\rangle\right\rangle-\left\langle\left\langle Q_{j}, Q_{i}\right\rangle\right\rangle\right)=0 .
$$

Следовательно, $\mathfrak{Q}_{\gamma, j} \subseteq \widetilde{\mathfrak{Q}}_{\gamma, j}$. Аналогичными рассуждениями получаем, что $\mathfrak{Q}_{\gamma, j} \supseteq \widetilde{\mathfrak{Q}}_{\gamma, j}$.

Определение 4. Линейное пространство $\mathfrak{Q}_{\gamma, j}$ назовем пространством квазирезонансных полиномов индекса $j \in \mathbb{Z}_{+}$. Через $\mathfrak{Q}_{\gamma, j}^{[k]}$ обозначим линейное пространство квазирезонансных квазиоднородных полиномов индекса $j$ обобщенной степени $k$ с каноническим весом $\gamma$.

Как видно из доказательства теоремы 1 , пространство $\mathfrak{Q}_{\gamma, j}$, в свою очередь, разбивается в прямую сумму ортогональных линейных подпространств $\mathfrak{Q}_{\gamma, j}^{[k]}$.

Покажем, как выглядят пространства $\mathfrak{Q}_{\gamma, j}$ для примеров 1 и 2 при условии, что канонический вес $\gamma$ выбран так, чтобы выполнялось неравенство $\chi>\delta$. В самих этих примерах нормальная форма от $\gamma$ не зависит, поэтому мы полагали $\gamma=(1,1)$. Теперь же нам нужен не совсем произвольный вес $\gamma$.

Обозначим через span переход к линейной оболочке.

Лемма 1. Пространства квазирезонанснљх полиномов индекса $j \in \mathbb{Z}_{+}$для невозмущенного гамильтониана $v^{m}, m \geqslant 2$, имеют вид

$$
\mathfrak{Q}_{\gamma, j}=\operatorname{span}\left\{u^{p} v^{q+j(m-1)}: p \in \mathbb{Z}_{+}, q=0, \ldots, m-2\right\} .
$$

Доказательство. Очевидно, что указанные пространства удовлетворяют всем условиям теоремы 1 .

Лемма 2. Пространства квазирезонансных полиномов индекса $j \in \mathbb{Z}_{+}$для невозмущенного гамильтониана $u^{l} v^{m}, l, m \geqslant 1, d=$ НОД $(l, m)$, имеют вид $\mathfrak{Q}_{\gamma, j}=\operatorname{span}\left\{u^{p+j(l-1)} v^{q+j(m-1)}:(p, q) \in I_{l, m}^{j}\right\}$, где $I_{l, m}^{j}$ обозначает множество пар $(p, q) \in \mathbb{Z}_{+}^{2}$, удовлетворлющих следующим условиям:

(i) $\prod_{i=0}^{j-1}(l q-m p-i(l-m)) \neq 0$;

(ii) $p=0, \ldots, l-2$ либо $p \geqslant l-1, q=0, \ldots, m-2$, либо $p=r l / d-1$, $q=r m / d-1, r \geqslant d$.

Замечание 1. В обоих рассматриваемых в леммах 1 и 2 случаях пространства квазирезонансных полиномов индекса $j \in \mathbb{Z}_{+}$представляются в виде $\mathfrak{Q}_{\gamma, j}=\widehat{H}_{\gamma}^{[\chi]^{j}}\left(\mathfrak{R}_{\gamma}\right)$.

Доказательство леммы 2. Достаточно показать, что указанные пространства удовлетворяют условиям теоремы 1.

Как было показано в примере $2, \widehat{H}_{\gamma}^{[\chi]^{*}}=\left(\partial^{l+m-2} / \partial u^{l-1} \partial v^{m-1}\right)(l(\partial / \partial v) v-$ $m(\partial / \partial u) u)$. Отсюда следует, что если $p+(j-k)(l-1) \in \mathbb{Z}_{+}$и $q+(j-k)(m-1) \in$ 
$\mathbb{Z}_{+}$, TO

$$
\begin{aligned}
\widehat{H}_{\gamma}^{[\chi]^{* k}}\left(u^{p+j(l-1)} v^{q+j(m-1)}\right) & =\frac{(p+j(l-1)) !(q+j(m-1)) !}{(p+(j-k)(l-1)) !(q+(j-k)(m-1)) !} \\
& \times\left(\prod_{i=1}^{k}(l q-m p+(i-j)(l-m))\right) u^{p+(j-k)(l-1)} v^{q+(j-k)(m-1)} .
\end{aligned}
$$

В остальных случаях $\widehat{H}_{\gamma}^{[\chi]^{* k}}\left(u^{p+j(l-1)} v^{q+j(m-1)}\right)=0$.

Полагая в $(6) k=j$ и $(p, q) \in I_{l, m}^{j}$, получаем соотношение $\left(4_{1}\right)$, а полагая в (6) $k=j+1$ и $(p, q) \in I_{l, m}^{j}-$ равенство $\left(4_{2}\right)$.

В случае $l=m$ оставшиеся условия теоремы 1 , очевидно, выполнены.

Пусть $l \neq m$. Допустим, что пространства $\operatorname{span}\left\{u^{p+j(l-1)} v^{q+j(m-1)}:(p, q) \in\right.$ $\left.\left.I_{l, m}^{j}\right\}\right)$ для различных $j \in \mathbb{Z}_{+}$не взаимно ортогональны. Тогда либо $r l / d+j(l-$ $1)=r^{\prime} l / d+j^{\prime}(l-1)$ и $r m / d+j(m-1)=r^{\prime} m / d+j^{\prime}(m-1)$, либо $r l / d-1+j(l-1)=$ $p+j^{\prime}(l-1)$ и $r m / d-1+j(m-1)=q+j^{\prime}(m-1)$, где $p=0, \ldots, l-2$ или $q=0, \ldots, m-2, r, r^{\prime} \geqslant d$ и $j \neq j^{\prime}$.

В первом случае, вычитая из первого равенства, умноженного на $m$, второе, умноженное на $l$, получаем $j(l-m)=j^{\prime}(l-m)$, что противоречит предположению $l \neq m, j \neq j^{\prime}$.

Во втором случае приходим к системе $\left(j^{\prime}-j\right)(l-1)=r l / d-p-1,\left(j^{\prime}-j\right)(m-1)$ $=r m / d-q-1$. При $j>j^{\prime}$ приходим к противоречию с предположением $p=$ $0, \ldots, l-2$ или $q=0, \ldots, m-2$, а при $j<j^{\prime}$, вычитая из первого равенства, умноженного на $m$, второе, умноженное на $l$, получаем $l q-m p-\left(j^{\prime}-j-1\right)(l-m)=0$, что противоречит предположению $(p, q) \in I_{l, m}^{j^{\prime}}$.

Наконец, как было отмечено в замечании 1,

$$
\bigoplus_{j=0}^{\infty} \operatorname{span}\left\{u^{p+j(l-1)} v^{q+j(m-1)}:(p, q) \in I_{l, m}^{j}\right\}=\bigoplus_{j=0}^{\infty} \widehat{H}_{\gamma}^{[\chi]^{j}}\left(\Re_{\gamma}\right)=\mathbb{K}[w]
$$

\section{§3. Нахождение неполной нормальной формы Белицкого}

3.1. Операция ๑. Рассмотрим гамильтониан $(1)$ с невозмущенной частью специального вида:

$$
H_{\gamma}^{[\chi]}(w)=H_{\gamma, 1}^{[\chi]}\left(u^{\prime}, v^{\prime}\right)+H_{\gamma, 2}^{[\chi]}\left(u^{\prime \prime}, v^{\prime \prime}\right) \quad(\chi>\delta, n \geqslant 2),
$$

где $u^{\prime}=\left(u_{1}, \ldots, u_{n^{\prime}}\right), v^{\prime}=\left(v_{1}, \ldots, v_{n^{\prime}}\right), u^{\prime \prime}=\left(u_{n^{\prime}+1}, \ldots, u_{n}\right), v^{\prime \prime}=\left(v_{n^{\prime}+1}, \ldots, v_{n}\right)$, $n^{\prime}<n$.

Введем следующие обозначения:

1) $\mathbb{K}\left[w^{\prime}\right]$ и $\mathbb{K}\left[w^{\prime \prime}\right]$ - алгебры полиномов от переменных $w^{\prime}=\left(u^{\prime}, v^{\prime}\right)$ и $w^{\prime \prime}=$ $\left(u^{\prime \prime}, v^{\prime \prime}\right)$;

2) $\mathfrak{Q}_{\gamma, j}^{\prime} \subset \mathbb{K}\left[w^{\prime}\right]$ и $\mathfrak{Q}_{\gamma, j}^{\prime \prime} \subset \mathbb{K}\left[w^{\prime \prime}\right]$ - пространства квазирезонансных полиномов индекса $j$ для $H_{\gamma, 1}^{[\chi]}$ и $H_{\gamma, 2}^{[\chi]}$ соответственно. 
По теореме 1 для всяких полиномов $P^{\prime} \in \mathbb{K}\left[w^{\prime}\right]$ и $P^{\prime \prime} \in \mathbb{K}\left[w^{\prime \prime}\right]$ единственны разложения

$$
P^{\prime}=\sum_{j=0}^{\infty} P_{\gamma, j}^{\prime}, \quad P^{\prime \prime}=\sum_{j=0}^{\infty} P_{\gamma, j}^{\prime \prime} \quad\left(P_{\gamma, j}^{\prime} \in \mathfrak{Q}_{\gamma, j}^{\prime}, P_{\gamma, j}^{\prime \prime} \in \mathfrak{Q}_{\gamma, j}^{\prime \prime}\right) .
$$

Определим билинейную операцию $\odot: \mathbb{K}\left[w^{\prime}\right] \times \mathbb{K}\left[w^{\prime \prime}\right] \rightarrow \mathbb{K}[w]$ следующим образом:

$$
P^{\prime} \odot P^{\prime \prime}=\sum_{j=0}^{\infty} \sum_{i=0}^{j}(-1)^{i} \widehat{H}_{\gamma, 1}^{[\chi]^{* i}}\left(P_{\gamma, j}^{\prime}\right) \widehat{H}_{\gamma, 2}^{[\chi]}{ }^{* j-i}\left(P_{\gamma, j}^{\prime \prime}\right) .
$$

Теорема 2. Операчия $\odot$ задает канонический изоморфизм пространств

$$
\bigoplus_{j=0}^{\infty} \mathfrak{Q}_{\gamma, j}^{\prime} \otimes \mathfrak{Q}_{\gamma, j}^{\prime \prime} \cong \mathfrak{R}_{\gamma}
$$

Доказательство. Действуя оператором (2) на (7), получим

$$
\begin{aligned}
& \widehat{H}_{\gamma}^{[\chi]^{*}}\left(P^{\prime} \odot P^{\prime \prime}\right) \\
& =\sum_{j=0}^{\infty} \sum_{i=0}^{j}(-1)^{i}\left(\widehat{H}_{\gamma, 1}^{[\chi]^{* i+1}}\left(P_{\gamma, j}^{\prime}\right) \widehat{H}_{\gamma, 2}^{[\chi]^{* j-i}}\left(P_{\gamma, j}^{\prime \prime}\right)+\widehat{H}_{\gamma, 1}^{[\chi]^{* i}}\left(P_{\gamma, j}^{\prime}\right) \widehat{H}_{\gamma, 2}^{[\chi]^{* j-i+1}}\left(P_{\gamma, j}^{\prime \prime}\right)\right) \\
& =\sum_{j=0}^{\infty} P_{\gamma, j}^{\prime} \widehat{H}_{\gamma, 2}^{[\chi]^{* j+1}}\left(P_{\gamma, j}^{\prime \prime}\right)+(-1)^{j} \widehat{H}_{\gamma, 1}^{[\chi]^{* j+1}}\left(P_{\gamma, j}^{\prime}\right) P_{\gamma, j}^{\prime \prime}=0 .
\end{aligned}
$$

Последнее равенство следует из определения квазирезонансных полиномов индекса $j$ (см. теорему 1 и определение 4).

Таким образом, полиномы вида (7) и их линейные комбинации - резонансные полиномы.

Пусть $Q \in \mathbb{K}[w]$ ортогонален всем полиномам вида (7). Тогда

$$
\begin{gathered}
\left\langle\left\langle Q, P^{\prime} \odot P^{\prime \prime}\right\rangle\right\rangle=\sum_{j=0}^{\infty} \sum_{i=0}^{j}(-1)^{i}\left\langle\left\langle\widehat{H}_{\gamma, 1}^{[\chi]^{i}} \widehat{H}_{\gamma, 2}^{[\chi]}{ }^{j-i}(Q), P_{\gamma, j}^{\prime} P_{\gamma, j}^{\prime \prime}\right\rangle\right\rangle=0 \\
\left(P^{\prime} \in \mathbb{K}\left[w^{\prime}\right], P^{\prime \prime} \in \mathbb{K}\left[w^{\prime \prime}\right]\right) .
\end{gathered}
$$

Последнее эквивалентно тому, что для каждого $j \in \mathbb{Z}_{+}$имеет место равенство

$$
\sum_{i=0}^{j}(-1)^{i} \widehat{H}_{\gamma, 1}^{[\chi]^{i}} \widehat{H}_{\gamma, 2}^{[\chi]^{j-i}}(Q)=\widehat{H}_{\gamma, 1}^{[\chi]}{ }^{j+1}\left(Q^{\prime}\right)+\widehat{H}_{\gamma, 2}^{[\chi]^{j+1}}\left(Q^{\prime \prime}\right),
$$

для некоторых полиномов $Q^{\prime}, Q^{\prime \prime} \in \mathbb{K}[w]$. Это возможно тогда и только тогда, когда

$$
Q=\widehat{H}_{\gamma, 1}^{[\chi]}\left(Q^{\prime}\right)+\widehat{H}_{\gamma, 2}^{[\chi]}\left(Q^{\prime}\right) \in \widehat{H}_{\gamma}^{[\chi]}(\mathbb{K}[w]),
$$

т. е. когда $Q$ ортогонально пространству резонансных полиномов. Отсюда вытекает, что всякий резонансный полином - это линейная комбинация полиномов вида (7), а значит,

$$
\mathfrak{R}_{\gamma}=\operatorname{span}\left\{P^{\prime} \odot P^{\prime \prime}: P^{\prime} \in \mathbb{K}\left[w^{\prime}\right], P^{\prime \prime} \in \mathbb{K}\left[w^{\prime \prime}\right]\right\} .
$$


Пусть $\left\{e_{j, k}^{\prime}\right\},\left\{e_{j, l}^{\prime \prime}\right\}\left(j \in \mathbb{Z}_{+}, k, l \in \mathbb{N}\right)$ - базисы пространств $\mathfrak{Q}_{\gamma, j}^{\prime}$ и $\mathfrak{Q}_{\gamma, j}^{\prime \prime}$ соответственно. Тогда $\left\{e_{j, k}^{\prime} \otimes e_{j, l}^{\prime \prime}\right\}-$ базис в $\mathfrak{Q}_{\gamma, j}^{\prime} \otimes \mathfrak{Q}_{\gamma, j}^{\prime \prime}$. Из (7) и (9) следует, что элементы $\left\{e_{j, k}^{\prime} \odot e_{j, l}^{\prime \prime}\right\}$ порождают пространство $\mathfrak{R}_{\gamma}$. Докажем, что они линейно независимы. Допустим, что $\sum_{k, l=1}^{\infty} c_{k, l} e_{j, k}^{\prime} \odot e_{j, l}^{\prime \prime}=0$ в предположении, что отлично от нуля лишь конечное число $c_{k, l}$. Тогда

$$
\sum_{k, l=1}^{\infty} c_{k, l} e_{j, k}^{\prime} \widehat{H}_{\gamma, 2}^{[\chi]^{* j}}\left(e_{j, l}^{\prime \prime}\right)=\sum_{k, l=1}^{\infty} c_{k, l} \sum_{i=1}^{j}(-1)^{i-1} \widehat{H}_{\gamma, 1}^{[\chi]}{ }^{* i}\left(e_{j, k}^{\prime}\right) \widehat{H}_{\gamma, 2}^{[\chi]^{* j-i}}\left(e_{j, l}^{\prime \prime}\right) \text {. }
$$

Поскольку справа отсутствуют слагаемые из $\mathfrak{Q}_{\gamma, j}^{\prime} \otimes \mathbb{K}\left[w^{\prime \prime}\right]$, а слева присутствуют только такие слагаемые, каждая из частей этого равенства должна равняться нулю.

В свою очередь, поскольку элементы $\widehat{H}_{\gamma, 2}^{[\chi]^{* j}}\left(e_{j, l}^{\prime \prime}\right)$ линейно независимы, левая часть может равняться нулю только при равенстве всех $c_{k, l}$ нулю.

Таким образом, элементы $e_{j, k}^{\prime} \odot e_{j, l}^{\prime \prime}$ образуют базис пространства $\mathfrak{R}_{\gamma}$, что доказывает изоморфизм (8). Наконец, поскольку $\odot$ не зависит от выбора базиса в $\bigoplus_{j=0}^{\infty} \mathfrak{Q}_{\gamma, j}^{\prime} \otimes \mathfrak{Q}_{\gamma, j}^{\prime \prime}$, изоморфизм (8) является каноническим.

3.2. Невозмущенный гамильтониан вида $v_{1}^{m_{1}} \pm v_{2}^{m_{2}}$. Рассмотрим гамильтониан (1) с квазиоднородной невозмущенной частью

$$
H_{\gamma}^{[\chi]}(w)=v_{1}^{m_{1}} \pm v_{2}^{m_{2}} \quad\left(m_{1}, m_{2} \geqslant 2\right),
$$

где $\gamma$ - произвольный канонический вес, такой, что $\chi=\gamma_{3} m_{1}=\gamma_{4} m_{2}>\delta$.

Следствие 1. Неполная нормальная форма Белиикого, соответствующая гамильтониану (1) с невозмущенной частью (10), имеет вид

$$
\widetilde{H}(z)=y_{1}^{m_{1}} \pm y_{2}^{m_{2}}+\sum_{j=0}^{\infty} \sum_{q_{1}=0}^{m_{1}-2} \sum_{q_{2}=0}^{m_{2}-2} \widetilde{h}^{(p, q, j)} x_{1}^{p_{1}} y_{1}^{q_{1}+j\left(m_{1}-1\right)} \odot x_{2}^{p_{2}} y_{2}^{q_{2}+j\left(m_{2}-1\right)},
$$

где коэффициенты $\widetilde{h}$ произвольны и

$$
\begin{aligned}
& x_{1}^{p_{1}} y_{1}^{q_{1}+j\left(m_{1}-1\right)} \odot x_{2}^{p_{2}} y_{2}^{q_{2}+j\left(m_{2}-1\right)}=x_{1}^{p_{1}} y_{1}^{q_{1}} x_{2}^{p_{2}} y_{2}^{q_{2}} \\
& \times \sum_{i=0}^{j}(\mp 1)^{j-i} \frac{\left(q_{1}+j\left(m_{1}-1\right)\right) !\left(q_{2}+j\left(m_{2}-1\right)\right) !}{\left(q_{1}+(j-i)\left(m_{1}-1\right)\right) !\left(q_{2}+i\left(m_{2}-1\right)\right) !}\left(m_{1} x_{1} y_{2}^{m_{2}-1}\right)^{i}\left(m_{2} x_{2} y_{1}^{m_{1}-1}\right)^{j-i} .
\end{aligned}
$$

Доказательство. Утверждение непосредственно следует из леммы 1 и теоремы 2 .

Замечание 2. Несмотря на то, что при $m_{1}, m_{2}=2$ в условиях следствия 1 предполагается, что возмущение гамильтониана (1) начинается с членов четвертой степени, формула (11) дает правильный результат и при наличии кубических членов в возмущении:

$$
\widetilde{H}(z)=y_{1}^{2} \pm y_{2}^{2}+\sum_{p_{1}+p_{2}+2 j \geqslant 3} \widetilde{h}^{\left(p_{1}, p_{2}, j\right)} x_{1}^{p_{1}} x_{2}^{p_{2}}\left(m_{1} x_{1} y_{2} \mp m_{2} x_{2} y_{1}\right)^{j} .
$$

Действительно, каждый из сомножителей под знаком суммы в правой части формулы (12) является интегралом линейной гамильтоновой системы, сопряженной к линейной системе, порожденной невозмущенным гамильтонианом 
$y_{1}^{2} \pm y_{2}^{2}$. Отсюда вытекает, что неполная нормальная форма Белицкого имеет вид (12).

3.3. Невозмущенный гамильтониан вида $v_{1}^{m_{1}} \pm \boldsymbol{u}_{2}^{l_{2}} \boldsymbol{v}_{2}^{m_{2}}$. Рассмотрим гамильтониан (1) с квазиоднородной невозмущенной частью

$$
H_{\gamma}^{[\chi]}(w)=v_{1}^{m_{1}} \pm u_{2}^{l_{2}} v_{2}^{m_{2}} \quad\left(m_{1} \geqslant 2, l_{2}, m_{2} \geqslant 1\right)
$$

где $\gamma$ - произвольный канонический вес, такой, что $\chi=\gamma_{3} m_{1}=\gamma_{2} l_{2}+\gamma_{4} m_{2}>\delta$.

Следствие 2. Неполная нормальная форма Белицкого, соответствующая гамильтониану (1) с невозмущенной частью (13), имеет вид

$$
\begin{aligned}
& \tilde{H}(z)=y_{1}^{m_{1}} \pm x_{2}^{l_{2}} y_{2}^{m_{2}} \\
& \quad+\sum_{j=0}^{\infty} \sum_{q_{1}=0}^{m_{1}-2} \sum_{\left(p_{2}, q_{2}\right) \in I_{l_{2}, m_{2}}^{j}} \widetilde{h}^{(p, q, j)} x_{1}^{p_{1}} y_{1}^{q_{1}+j\left(m_{1}-1\right)} \odot x_{2}^{p_{2}+j\left(l_{2}-1\right)} y_{2}^{q_{2}+j\left(m_{2}-1\right)}
\end{aligned}
$$

где коэфбиииенты $\widetilde{h}$ произвольны, множества $I_{l, m}^{j}$ определены в лемме 2 и

$$
\begin{aligned}
x_{1}^{p_{1}} y_{1}^{q_{1}+j\left(m_{1}-1\right)} \odot x_{2}^{p_{2}+j\left(l_{2}-1\right)} y_{2}^{q_{2}+j\left(m_{2}-1\right)}=x_{1}^{p_{1}} y_{1}^{q_{1}} x_{2}^{p_{2}} y_{2}^{q_{2}} \\
\times \sum_{i=0}^{j} \frac{( \pm 1)^{j-i}\left(p_{2}+j\left(l_{2}-1\right)\right) !\left(q_{1}+j\left(m_{1}-1\right)\right) !\left(q_{2}+j\left(m_{2}-1\right)\right) !}{\left(p_{2}+i\left(l_{2}-1\right)\right) !\left(q_{1}+(j-i)\left(m_{1}-1\right)\right) !\left(q_{2}+i\left(m_{2}-1\right)\right) !} \\
\quad \times\left(\prod_{k=1}^{j-i}\left(l_{2} q_{2}-m_{2} p_{2}+k\left(l_{2}-m_{2}\right)\right)\right)\left(m_{1} x_{1} x_{2}^{l_{2}-1} y_{2}^{m_{2}-1}\right)^{i} y_{1}^{\left(m_{1}-1\right)(j-i)} .
\end{aligned}
$$

Доказательство. Утверждение непосредственно следует из лемм 1, 2 и теоремы 2 .

Замечание 3. Случай $m_{1}=2, l_{2}, m_{2}=1$ не подпадает под условия следствия 2. Тем не менее формула (14) и для него дает правильный результат:

$$
\widetilde{H}(z)=y_{1}^{2} \pm x_{2} y_{2}+\sum_{p_{1}+2 p_{2} \geqslant 3} \widetilde{h}^{\left(p_{1}, p_{2}\right)} x_{1}^{p_{1}}\left(x_{2} y_{2}\right)^{p_{2}}
$$

Действительно, всякий полиномиальный интеграл линейной гамильтоновой системы, сопряженной к линейной системе, порожденной невозмущенным гамильтонианом $y_{1}^{2} \pm x_{2} y_{2}$, является полиномом от $x_{1}$ и $x_{2} y_{2}$. Отсюда вытекает, что неполная нормальная форма Белицкого имеет вид (15).

3.4. Невозмущенный гамильтониан вида $h_{1} u_{1}^{l_{1}} v_{1}^{m_{1}}+h_{2} u_{2}^{l_{2}} v_{2}^{m_{2}}$. Рассмотрим гамильтониан (1) с квазиоднородной невозмущенной частью

$$
H_{\gamma}^{[\chi]}(w)=h_{1} u_{1}^{l_{1}} v_{1}^{m_{1}}+h_{2} u_{2}^{l_{2}} v_{2}^{m_{2}} \quad\left(h_{i} \neq 0, l_{i}, m_{i} \geqslant 1\right)
$$

где $\gamma$ - произвольный канонический вес, такой, что $\chi=\gamma_{1} l_{1}+\gamma_{3} m_{1}=\gamma_{2} l_{2}+$ $\gamma_{4} m_{2}>\delta$. 
Следствие 3. Неполная нормальная форма Белицкого, соответствующая гамильтониану (1) с невозмущенной частью (16), имеет вид

$$
\begin{aligned}
\widetilde{H}(z) & =h_{1} x_{1}^{l_{1}} y_{1}^{m_{1}}+h_{2} x_{2}^{l_{2}} y_{2}^{m_{2}} \\
& +\sum_{\substack{\left(p_{1}, q_{1}\right) \in I_{l_{1}, m_{1}}^{j},\left(p_{2}, q_{2}\right) \in I_{l_{2}, m_{2}}^{j}}} \widetilde{h}^{(p, q, j)} x_{1}^{p_{1}+j\left(l_{1}-1\right)} y_{1}^{q_{1}+j\left(m_{1}-1\right)} \odot x_{2}^{p_{2}+j\left(l_{2}-1\right)} y_{2}^{q_{2}+j\left(m_{2}-1\right)},
\end{aligned}
$$

где коэффициенты $\widetilde{h}$ произвольны, множества $I_{l, m}^{j}$ определены в лемме 2 и

$$
\begin{aligned}
& x_{1}^{p_{1}+j\left(l_{1}-1\right)} y_{1}^{q_{1}+j\left(m_{1}-1\right)} \odot x_{2}^{p_{2}+j\left(l_{2}-1\right)} y_{2}^{q_{2}+j\left(m_{2}-1\right)}=x_{1}^{p_{1}} y_{1}^{q_{1}} x_{2}^{p_{2}} y_{2}^{q_{2}} \\
& \times \sum_{i=0}^{j}(-1)^{i} \frac{\left(p_{1}+j\left(l_{1}-1\right)\right) !\left(p_{2}+j\left(l_{2}-1\right)\right) !\left(q_{1}+j\left(m_{1}-1\right)\right) !\left(q_{2}+j\left(m_{2}-1\right)\right) !}{\left(p_{1}+(j-i)\left(l_{1}-1\right)\right) !\left(p_{2}+i\left(l_{2}-1\right)\right) !\left(q_{1}+(j-i)\left(m_{1}-1\right)\right) !\left(q_{2}+i\left(m_{2}-1\right)\right) !} \\
& \times\left(\prod_{k=1}^{i}\left(l_{1} q_{1}-m_{1} p_{1}+k\left(l_{1}-m_{1}\right)\right)\right) \\
& \left(\prod_{k^{\prime}=1}^{j-i}\left(l_{2} q_{2}-m_{2} p_{2}+k^{\prime}\left(l_{2}-m_{2}\right)\right)\right) \\
& \times\left(\bar{h}_{1} x_{2}^{l_{2}-1} y_{2}^{m_{2}-1}\right)^{i}\left(\bar{h}_{2} x_{1}^{l_{1}-1} y_{1}^{m_{1}-1}\right)^{j-i} .
\end{aligned}
$$

Доказательство. Утверждение непосредственно следует из леммы 2 и теоремы 2 .

Замечание 4. Случай $l_{1}, m_{1}, l_{2}, m_{2}=1$ не подпадает под условия следствия 3. Тем не менее, если $h_{1}$ и $h_{2}$ рационально несоизмеримы, формула (17) и для него дает правильный результат:

$$
\widetilde{H}(z)=h_{1} x_{1} y_{1}+h_{2} x_{2} y_{2}+\sum_{p_{1}+p_{2} \geqslant 2} \widetilde{h}^{\left(p_{1}, p_{2}\right)}\left(x_{1} y_{1}\right)^{p_{1}}\left(x_{2} y_{2}\right)^{p_{2}} .
$$

Таким образом, формула (17) обобщает классический результат Биркгофа $[4$, III.8] в случае двух степеней свободы.

\section{ЛитературА}

[1] Г. Р. Белицкий, Инвариантные нормалъные формы формалъных рядов, Функц. анализ и его прилож., 13:1 (1979), 59-60.

[2] Г. Р. Белицкий, Нормальные формы относительно действия группы в пространстве, Изв. АН СССР, сер. матем., 41:5 (1977), 1053-1063.

[3] В. В. Басов, А. С. Ваганян, Нормальные формы гамильтоновых систем, Дифференциальные уравнения и процессы управления, 2010, № 4, 86-107 (эл. журнал http://www.math.spbu.ru/diffjournal).

[4] G. D. Birkhoff, Dynamical Systems, Colloquium Publications, vol. 9, Amer. Math. Soc., Providence, 1927.

Санкт-Петербургский государственный университет e-mail: vlvlbasov@rambler.ru

Поступило в редакцию

Санкт-Петербургский государственный университет 21 апреля 2013 г.

e-mail: armay@yandex.ru 